TESTS FOR PROPERNESS IN PERIODIC

CONTROL OF FUNCTIONAL DIFFERENTIAL SYSTEMS

\title{
Fritz Colonius
}

Division of Applied Mathematics, Box F Brown University, Providence, RI 02912

\section{Summary}

A fundamental problem in optimal periodic control may be formulated as follows: Suppose one has an optimal steady state $x^{0}$ corresponding to a constant control $u^{0}$. Can performance be improved by allowing for trajectories $x$ and controls $u$ being periodic with some common period $\tau>0$ ? If this is the case, the problem is called proper. For systems governed by ordinary differential equations the so called I-criterion is a second order variational test for (local) properness. It has been proposed by Bittanti, Fronza, and Guarbadassi [1] and proven by Bernstein and Gilbert [3]; the most general version can be found in Bernstein [2]. Watanabe, Nishimura and Matsubara [12] gave a variant of the I-criterion ('singular control test' or 'infinite frequency I-criterion') which tests properness for sufficiently high frequencies and requires significantly less computational effort.

The I-criterion is of some relevance in chemical engineering and aircraft flight performance optimization. (cp. Sincic and Bailey [9], Speyer [11] and the survey papers by Matsubara, Nishimura, Watanabe, Onogi [7] and Noldus [8]).

This paper presents a generalization to functional differential systems of the $\pi$-criterion and its "highfrequency" variant.

\section{Problem Formulation}

We consider the following optimal periodic control problem:

$$
\begin{aligned}
& \text { (OPC) Minimize } \frac{1}{\tau} \int_{0}^{\tau} g(x(t), u(t)) d t \\
& \text { s.t. (2.1) } \dot{x}(t)=f(x(t), u(t)) \text { a.a. } t \in[0, \tau] \\
& (2.2) x_{0}=x_{\tau}
\end{aligned}
$$

where $\tau>0$ is fixed, $x_{t}(s):=x(t+s) \in \mathbb{R}^{n}, s \in[-h, 0]$, $u(t) \in \mathbb{R}^{m}$, and $h>0$ is the length of the delay. The maps $f: C\left(-h, 0 ; \mathbb{R}^{n}\right) \times \mathbb{R}^{m} \rightarrow \mathbb{R}^{n}$ and $g: \mathbb{R}^{n} \times \mathbb{R}^{\mathrm{m}} \rightarrow \mathbb{R}$ are assumed to be twice continuously Fréchet differentiable.

The controls $u$ are taken in $L_{\infty}\left(0, \tau ; \mathbb{R}^{\mathbb{M}}\right)$.

In this problem formulation, the periodicity condition for $x$ is incorporated into (2.2). Observe that the finite dimensional condition

$$
x(0)=x(\tau)
$$

does not guarantee periodic extendability of $x$ to a solution of (2.1) for $t \geq 0$ (with periodic extension of $u)$. Instead we have to consider the constraint (2.2) involving the states $x_{0}$ and $x_{\tau}$. We impose the following
Assumption: For every initial function $\mathrm{x}_{0}=\psi \in C\left(-\mathrm{h}, 0 ; \mathbb{R}^{\mathrm{n}}\right)$ and every control $u \in \mathrm{L}_{\infty}\left(0, T ; \mathbb{R}^{\mathrm{m}}\right)$, equation (2.1) has a unique absolutely continuous solution $x$.

The optimal steady state problem corresponding to (OPC) has the following form.

(oss) Minimize $g(x, u)$ $x \in \mathbb{R}^{n}, u \in \mathbb{R}^{m}$

s.t. (2.3) $0=f(\bar{x}, u)$

where $\bar{x} \in C\left(-h, 0 ; \mathbb{R}^{n}\right)$ is the constant function $\overline{\mathrm{x}}(\mathrm{s}):=\mathrm{x}$.

We are interested in the property specified by the following definition.

Definition: Let $\left(x^{0}, u^{0}\right) \in \mathbb{R}^{n} \times \mathbb{R}^{m}$ be an optimal solution of (OSS). Then $\left(x^{0}, u^{0}\right)$ is called locally proper if for all $\varepsilon>0$ there exist $x$ and $u$ satisfying (2.1) and (2.2) with $\|x-\vec{x}\|_{\infty}<\varepsilon,\|u-\vec{u}\|_{\infty}<\varepsilon$ and $\frac{1}{\tau} \int_{0}^{\tau} g(x(t), u(t)) d t<g\left(x^{0}, u^{0}\right)$.

\section{Tests for Properness}

Let $\left(x^{0}, u^{0}\right) \in \mathbb{R}^{n} \times \mathbb{R}^{m}$ be a steady state, i.e., satisfy (2.3). Then we can linearize the system equation $(2.1)$ around $(\vec{x}, \vec{u})$ and find

$$
\dot{x}(t)=I x_{t}+B u(t), a \cdot a \cdot t \in[0, \tau]
$$

where

$$
\begin{aligned}
& L:=D_{1} f\left(\vec{x}^{0}, u^{0}\right): C\left(-h, 0 ; \mathbb{R}^{n}\right) \rightarrow \mathbb{R}^{n} \\
& B:=D_{2} f\left(\bar{x}^{0}, u^{0}\right) \in \mathbb{R}^{n \times m} .
\end{aligned}
$$

The corresponding characteristic matrix $\Delta(z)$ is given by

$$
\Delta(z):=z I-L\left(e^{z \cdot}\right) \quad z \in \mathbb{C},
$$

where $e^{z}$ denotes the function $\exp (z \theta), \theta \in[-\mathrm{h}, 0]$, and $I$ is the $n \times n$ unit matrix. Introduce the function $\mathrm{H}: \mathrm{C}\left(-\mathrm{h}, 0 ; \mathbb{R}^{\mathrm{n}}\right) \times \mathbb{R}^{\mathrm{m}} \times \mathrm{R}^{\mathrm{In}} \rightarrow \mathbb{R}$

$$
H(\psi, u, \lambda):=g(\psi(0), u)+\lambda^{t} f(\psi, u) \text {. }
$$

Then the following expressions exist (here $j:=\sqrt{-1}$ ). 


$$
\begin{aligned}
P(\omega): & =D_{1} D_{1} H\left(\vec{x}^{0}, u^{0}, \lambda\right)\left(e^{j \omega \cdot}, e^{-j \omega \cdot}\right) \\
Q(\omega) & :=D_{2} D_{1} H\left(\vec{x}^{0}, u^{0}, \lambda\right)\left(e^{j \omega \cdot}\right) \\
R & :=D_{2} D_{2} H\left(\vec{x}^{0}, u^{0}, \lambda\right) .
\end{aligned}
$$

We identify $P(\omega), Q(\omega)$, and $R$ with elements in $C^{n \times n}$, $\mathbb{C}^{\mathrm{n} \times \mathbb{m}}$, and $\mathbb{R}^{\mathbb{m} \times \mathbb{m}}$, respectively. Define for $\omega \in \mathbb{R}$ the complex mxm - matrix $T(\omega)$ by

$$
\begin{aligned}
\because(\omega):=B & \Delta^{-I}(-j \omega)^{t} P(\omega) \Delta^{-1}(j \omega) B \\
& +Q(-\omega)^{t} \Delta^{-1}(j \omega) B+B^{t} \Delta^{-1}(-j \omega)^{t} Q(\omega) \\
& +R .
\end{aligned}
$$

The matrix $\Pi(\omega)$ is Hermitian. We assume that the following normality condition for (OSS) is satisfied:

$$
\left.\mathbb{R}^{n}=i D_{I} f\left(\bar{x}^{0}, u^{0}\right) \bar{x}\left|x \in \mathbb{R}^{n} ;+i D_{2} f\left(\bar{x}^{0}, u^{0}\right) u\right| u \in \mathbb{R}^{m}\right\} .
$$

Then the following I-Criterion is valid:

Theorem 1: Suppose that $\left(x^{0}, u^{0}\right) \in \mathbb{R}^{n} \times \mathbb{R}^{\mathbb{R}}$ is an optimal solution of (OSS) and that $j k w, k \in \mathbb{Z}$, is not a zero of $L(z)$, for $\omega=2 \pi / \tau$. .

(i) Then there exists $\lambda \in \mathbb{R}^{n}$ such that

$$
\left\{\begin{array}{l}
0=D_{1} H\left(\vec{x}^{0}, u^{0}, \lambda\right) \\
0=D_{2} H\left(\vec{x}^{0}, u^{0}, \lambda\right) .
\end{array}\right.
$$

(ii) Let $\lambda \in \mathbb{R}^{n}$ satisfy (i) and suppose that there is $\eta \in \mathbb{R}^{\mathrm{m}}$ with

$$
\eta^{t} \Pi(\omega) r_{1}<0
$$

Then $\left(x^{0}, u^{0}\right)$ is locally proper. Suppose that $\Delta(z)$ has no zeros in the closed right half plane $z \in C: \operatorname{Re} z>0\}$. Then a high frequency variant of this T-criterion can be obtained through the following series expansion of $\Pi(\omega)$ : Let

$$
A(\omega):=L\left(e^{j \omega \cdot}\right)
$$

and define

$$
\begin{aligned}
& R_{0}:=R \\
& R_{k}:=\left[\begin{array}{ll}
Q^{t}(-\omega) & B^{t}
\end{array}\right]\left[\begin{array}{lc}
A(\omega) & 0 \\
-P(\omega)(j \omega) & -A^{t}(-\omega)
\end{array}\right]^{k-1}\left[\begin{array}{c}
B \\
-Q(\omega)
\end{array}\right] \text {. }
\end{aligned}
$$

Then

$$
I(\omega)=\sum_{k=0}^{\infty}(j \omega)^{-k_{R_{k}}(\omega)} .
$$

and one can prove the following high-frequency I-Criterion.

Theorem 2: There exists $\omega_{0}>0$ such that for alI $\omega \geq \omega_{0}$ either of the following conditions implies that the optimal steady state $\left(\mathrm{x}^{0}, \mathrm{u}^{0}\right)$ is locally proper: (i) For all $k=0,1, \ldots 2,-1$ one has $R_{k}(\omega)=0$ and there exists $r \in \mathbb{R}^{m}$ such that $(-1)^{\ell} r_{i} t_{2 l}(\omega) \eta<0$. (ii) For all $k=0,1, \ldots, 2 \hat{i}$ one has $R_{k}(\omega)=0$ and there exists $\eta \in \mathbb{R}^{\mathbb{M}}$ such that

$$
(-1)^{x+1} j n^{t} R_{2 x+1}(\omega) n<0 \text {. }
$$

Remark: The system equation (2.1) also allows the delays to depend on state and time. Manitius [6] computed the corresponding Frechet derivatives. Sincic, Bailey [10] use the same formulae for the derivatives, and indicate the formulae for the second derivatives.
They give a (formal) proof of the $J$-Criterion in this rase.

\section{References}

[1] S. Bittanti, G. Fronza, and G. Guarbadassi, Periodic Control: A frequency domain approach, IEEE Irans. Aut. Control AC-18 (1973), 33-38.

[2] D. S. Bernstein, Control constraints, abnormality, and improved performance by periodic control, submitted for publication.

[3] D. S. Bernstein, E. G. Gilbert, Optimal periodic contro1: The Ii-Test revisited, IEEE Trans. Aut. Control, AC-25 (1980), 673-684.

[4] F. Colonius, Optimality for periodic control of functional differential systems, Report No. 36-1984, Mathematisches Institut der Universität Graz, Graz 1984, submitted for publication.

[5] F. Colonius, The high frequency J-Criterion for retarded systems, Report No. 37-1984, Mathematisches Institut der Lniversität, Graz 1984, submitted for publication.

[6] A. Manitius, on the optimal control of systems with a delay depending on state, control and time, CRM-449, Université de Montreal 1974.

[7] M. Matsubara, Y. Nishimura, N. Watanabe, K. Onogi, Periodic Control Theory and applications, Research Reports of Automatic Control Laboratory, Vol. 28, Faculty of Engineering, Nagoya University, 1981.

[8] E. Noldus, A survey of periodic control of continuous systems, Journal A, 16 (1975), 11-16.

[9] D. Sincic, J. E. Balley, Analytical Optimization and sensitivity analysis of forced periodic chemical processes, Chem. Eng. Sci., 35 (1980), 11531165.

[10] D. Sincic, J. E. Bailey, Optimal periodic control of variable time-delay systems, Int. J. Control, 27 (1978), 547-555.

[11] J. L. Speyer, Non-optimality of steady state cruise for aircraft, AIAA Journa1, 14 (1976), 1604-1610.

[12] N. Watanabe, Y. Nishimura, M. Matsubara, Singular Control Test for optimal periodic control problems, IEEE Trans. Aut. Control, AC-21 (1976), $609-610$. 